\title{
STUDIO COMPARATIVO DEI MICROSISMI REGISTRATI A ROMA ED A TRIESTE
}

\section{Carlo Morelli}

1. Premessa. -- È noto come il problema dei microsismi sia oggi ancora per molti aspetti un problema aperto in Sismologia, sia per quanto ne riguarda la genesi, ed in particolare il suo meccanismo, sia per quanto si riferisce alla loro propagazione ed alla natura fisica. Sono stati pubblicati in altri lavori i risultati delle ricerche effettuate all'Osservatorio Geofisico di Trieste sul problema in generale $\left({ }^{1}\right)$, nonché sulle cause $\left({ }^{2}\right)$ e sulla natura fisica $\left({ }^{3}\right)$ dei microsismi. Qui ci proponiamo di vedere quali elementi si possano dedurre da un esame comparativo dei microsismi registrati in due Stazioni diverse.

In Italia le Stazioni sismiche sono ormai abbastanza numerose, ma strumenti a registrazione fotografica, cioè con ingrandimento sufficiente per mettere bene in evidenza le micropulsazioni del suolo che qui si vogliono studiare, sono in funzione soltanto a Roma ed a Trieste: Galitzin-Wilip e Galitzin-ING a Roma, Alfani a Trieste. Le costanti strumentali, per l'intervallo di tempo più sotto specificato, sono le seguenti:

\begin{tabular}{|c|c|c|c|c|c|c|c|c|}
\hline \multicolumn{9}{|c|}{ ROMA } \\
\hline Sismografo & Comp. & $\mathrm{T}_{\mathrm{g}}$ & $T_{a}$ & $v_{n}$ & K & $\mu^{2}$ & $A_{1}$ & $l_{\mathrm{r}}$ \\
\hline Galitzin-Wilip & N-S & 9,6 & 9,26 & 1480 & 158,97 & 0.00126 & 100 & 114,9 \\
\hline Galitzin-Wilip & E.W & 9,8 & 9,6 & 1330 & 142,25 & $-0,00126$ & 100 & 115,4 \\
\hline Galitzin-Wilip & Z & 10,1 & 9,7 & 1125 & 155,56 & 0,00755 & 100 & 147,9 \\
\hline
\end{tabular}

TRIESTE

\begin{tabular}{|c|c|c|c|c|c|}
\hline Sismografo & Comp. & Tu & $V_{0}$ & $v$ & Smorzam. \\
\hline Alfani & E.W & 12,5 & 1700 & aperiod. & magnetico \\
\hline Alfani & N.S & 10,5 & 1400 &, &,
\end{tabular}


Come si vede, i periodi lelle componenti orizzontali sono dello stesso ordine di grandezza. A Trieste manca purtroppo ancora la componente verticale, in quanto l'origrinale era con molla di acciaio, e quindi troppo sensibile ad ogni minima variazione di temperatura. Nel 1949 è stato possibile acquistare una molla di elinvar, che però neppure è sufficiente ad eliminare del tutto gli effetti dovuti alle variazioni di temperatura. $\grave{E}$ stato allora costruito un termocompensatore, cbe però è ancora in fase di sperimentazione. Il confronto quindi sarà basato sulle registrazioni delle due componenti orizzontali.

L'intervallo di tempo considerato va dal febbraio 1948 al febbraio 1919. In entrambe le Stazioni le letture essendo effettuate, secondo la convenzione internazionale, alle $0^{\mathrm{h}}, 6^{\mathrm{h}}, 12^{\mathrm{h}}$ e $18^{\mathrm{h}}(T M G)$, il confronto è stato effettuato per tutte le giornate a queste ore, calcolando il rapporto fra le ampiezze del moto vero del suolo (espresse in $\mu$ a Roma ed a Trieste per onde di identico periodo (espresso in sec). Le ampiezze di Roma sono state dedotte dal Bollettino sismico mensile pubblicato da quella Stazione; inoltre è stato tenuto conto delle eventuali diversità di periodo e di ogni altra particolarità delle registrazioni, secondo quanto più avanti esporremo.

Il confronto di cui sopra trova la sua giustificazione nel fatto che il periodo dei microsismi è anzitutto funzione della distanza (del centro della perturbazione dalla Stazione registratrice), anzi è spesso caratteristico per una determinata zona ( $\left.{ }^{1}\right)$ : per esempio, per Trieste, esso può arrivare fino a 4 sec per cicloni sul Mediterraneo, mentre periodi maggiori corrispondono in generale a cicloni sull'Atlantico.

2. Rapporto delle ampiezze a Roma ed a Trieste. - I risultati del confronto sono riportati nel quadro seguente, in cui in corrispondenza a valori del periodo $T$ di 0,5 in $0,5 \mathrm{sec}$, figurano per le due componenti E-W e N-S: il numero dei casi presi in considerazione e la media del rapporto fra le ampiezze a Roma, $A_{\mathrm{r}}$, ed a Trieste, $A_{\mathrm{t}}$. con i rispettivi errori medi $M$; nell'ultima colonna è riportata la media (ponderata) fra le due componenti.

L'andamento del rapporto delle ampiezze fra Roma e Trieste è riportato graficamente in fig. $l$.

L'esame della tabella, ma soprattutto del grafico, mette anzitutto in evidenza una chiara prevalenza delle ampiezze registrate a Roma, che si attenua solo per i periodi più lunghi, corrispondenti a cicloni a N.W dell'Islanda.

Per spiegare questo fatto, bisogna anzitutto considerare la diversa situazione geologica locale delle Stazioni di Roma e di Trie- 


\begin{tabular}{|c|c|c|c|c|c|c|c|c|c|c|c|}
\hline$T$ & $\dot{\vec{g}}$ & n. & $A_{r} / A_{t}$ & M & Media & $\mathbf{T}$ & نे & n. & $A_{r} / A_{t}$ & M & Media \\
\hline 2,0 & $\begin{array}{l}\mathbf{E} \\
\mathbf{N}\end{array}$ & $\begin{array}{l}57 \\
41\end{array}$ & $\begin{array}{l}4,74 \\
3,43\end{array}$ & $\begin{array}{r}0,58 \\
0,55\end{array}$ & $4,08-0,66$ & 5,5 & $\begin{array}{l}\mathbf{E} \\
\mathbf{N}\end{array}$ & $\begin{array}{r}109 \\
71\end{array}$ & $\begin{array}{l}2,84 \\
2,82\end{array}$ & $\begin{array}{l}0,19 \\
0,22\end{array}$ & $2,83-1-0,01$ \\
\hline 2,5 & $\begin{array}{l}\mathbf{E} \\
\mathbf{N}\end{array}$ & $\begin{array}{l}29 \\
19\end{array}$ & $\begin{array}{l}4,19 \\
4,49\end{array}$ & $\begin{array}{l}0,73 \\
0,87\end{array}$ & $4,29+0,14$ & 6,0 & $\begin{array}{l}\mathbf{E} \\
\mathbf{N}\end{array}$ & $\begin{array}{l}94 \\
58\end{array}$ & $\begin{array}{l}2,39 \\
2.77\end{array}$ & $\begin{array}{l}0,31 \\
0,24\end{array}$ & $2.64-0,18$ \\
\hline 3,0 & $\begin{array}{l}\mathbf{E} \\
\mathbf{N}\end{array}$ & $\begin{array}{l}41 \\
13\end{array}$ & $\begin{array}{l}4,43 \\
7,54\end{array}$ & $\begin{array}{l}0,53 \\
1,70\end{array}$ & $4,68+0,84$ & 6,5 & $\begin{array}{l}\mathbf{E} \\
\mathbf{N}\end{array}$ & $\begin{array}{l}49 \\
25\end{array}$ & $\begin{array}{l}2,26 \\
2,34\end{array}$ & $\begin{array}{l}0,23 \\
0,55\end{array}$ & $2,27-0,03$ \\
\hline 3,5 & $\begin{array}{l}\mathbf{E} \\
\mathbf{N}\end{array}$ & $\begin{array}{l}12 \\
-\end{array}$ & $\begin{array}{c}6,75 \\
-\end{array}$ & $\begin{array}{c}0,65 \\
-\end{array}$ & $6,75-0,65$ & 7,0 & $\begin{array}{l}\mathbf{E} \\
\mathbf{N}\end{array}$ & $\begin{array}{l}46 \\
18\end{array}$ & $\begin{array}{l}2.41 \\
2.19\end{array}$ & $\begin{array}{ll}0 & 22 \\
0,35\end{array}$ & $2,35+0,09$ \\
\hline 4,0 & $\begin{array}{l}\mathbf{E} \\
\mathbf{N}\end{array}$ & $\begin{array}{l}95 \\
34\end{array}$ & $\begin{array}{l}3,69 \\
2,88\end{array}$ & $\begin{array}{l}0,35 \\
0,35\end{array}$ & $3,28+0,40$ & 7,5 & $\begin{array}{l}\mathbf{E} \\
\mathbf{N}\end{array}$ & $\begin{array}{r}22 \\
7\end{array}$ & $\begin{array}{l}1,92 \\
1,38\end{array}$ & $\begin{array}{l}0,18 \\
0,20\end{array}$ & $2,65+0,27$ \\
\hline 4,5 & $\begin{array}{l}\mathbf{E} \\
\mathbf{N}\end{array}$ & $\begin{array}{l}94 \\
42\end{array}$ & $\begin{array}{l}2,90 \\
2,74\end{array}$ & $\begin{array}{l}0,82 \\
0,37\end{array}$ & $2,77+0,14$ & 8,0 & $\begin{array}{l}\mathbf{E} \\
\mathbf{N}\end{array}$ & $\begin{array}{r}23 \\
2\end{array}$ & $\begin{array}{l}1,96 \\
1,05\end{array}$ & $\begin{array}{l}0,21 \\
0.55\end{array}$ & $1,83+0,32$ \\
\hline 5,0 & $\begin{array}{l}\mathbf{E} \\
\mathbf{N}\end{array}$ & $\begin{array}{r}112 \\
63\end{array}$ & $\begin{array}{l}3,40 \\
3,52\end{array}$ & $\begin{array}{l}0,27 \\
0,33\end{array}$ & $3,46-1-0,06$ & $\begin{array}{l}8,5 \\
9,0\end{array}$ & $\begin{array}{l}\mathbf{E} \\
\mathbf{E}\end{array}$ & $\begin{array}{l}2 \\
2\end{array}$ & $\begin{array}{l}1,00 \\
0,95\end{array}$ & $\begin{array}{l}0,32 \\
0,26\end{array}$ & $\begin{array}{l}1,00+0,32 \\
0,95+0,26\end{array}$ \\
\hline
\end{tabular}

ste: mentre a Trieste i sismografi poggiano direttamente sul crostello di arenaria, fogliettata (flysch) ma consistente, spessa qualche centinaio di $\mathrm{m}$, cui fanno seguito i calcari, a Roma il terreno è di riporto, sovrastante al tufo litoide.

a) Si può quindi anzitutto concludere che il movimento registrato

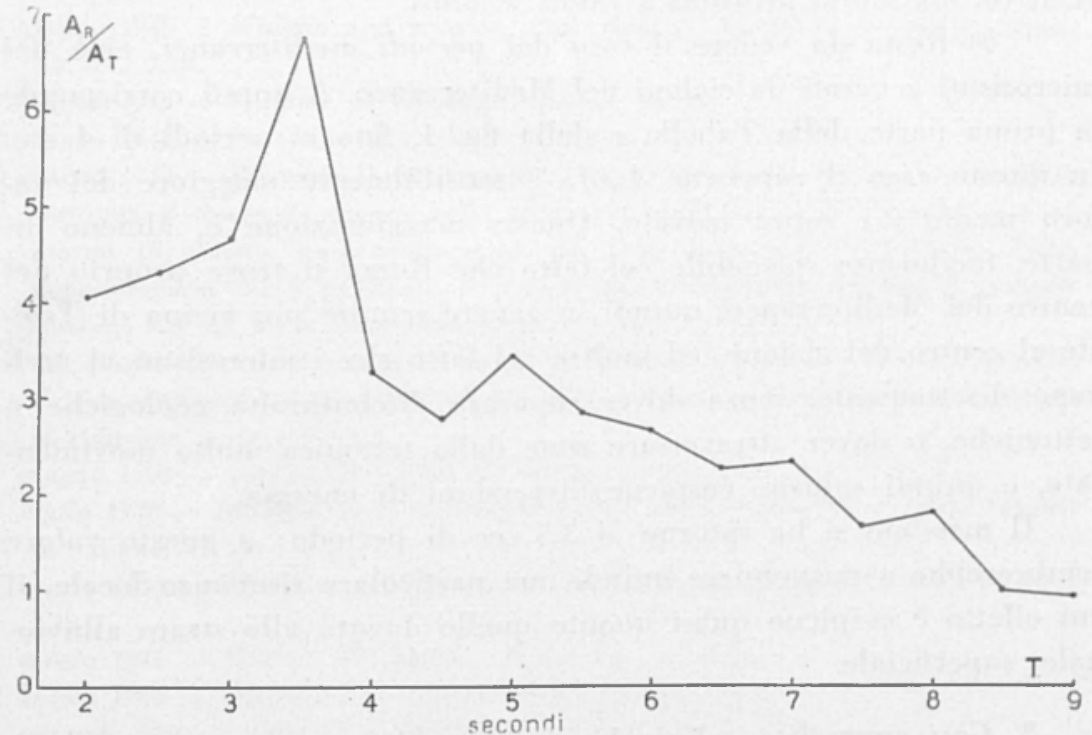

Fig. 1 - Rapporti fra le ampiezze dei microsismi di egual periodo a Roma ed a Trieste 
a Roma viene esaltato in maniera cospicua dalla coltre alluvionale su cui poggia la Stazione Sismica. Considerando soltanto i cicloni atlantici, che a priori dovrebbero produrre gli stessi effetti a Roma ed a Trieste, e assumendo per normale il valore di Trieste, si può ammettere che in conseguenza della situazione geologica superficiale, il fattore di amplificazione per le ampiezze a Roma corrispondenti a periodi fra 5 e 8 sec (microsismi sicuramente atlantici sia a Roma che a Trieste) sia dell'ordine di 2,1 (i valori del rapporto corrispondenti ai periodi di 8,5 e 9,0 sec non sono stati considerati, perché hasati su troppo pochi casi).

Sempre assumendo come riferimento l'ampiezza dei microsismi a Trieste fatta uguale ad 1 , il fattore di amplificazione per alcune altre Stazioni italiane risulta dunque $\left({ }^{4}\right)$ :

$\begin{array}{cccccc}\text { Roma } & \text { Catania } & \text { Padova } & \text { Salò } & \text { Trieste } & \text { Messina } \\ 2,1 & 2,1 & 1,7 & 1,1 & 1,0 & 0,9\end{array}$

'Tenendo conto che a Messina i sismografi poggiano direttamente sulla roccia sottostante come a Trieste, e che a Salò il terreno di riporto è di piccolo spessore, sembra lecito considerare giustificato il valore unitario attribuito al fattore di amplificazione a Trieste e Mes. sina, e quindi ritenere i valori sopra riportati validi non solo in senso relativo, ma molto prossimi a valori assoluti.

b) Resta da vedere il caso dei periodi mediterranei, cioè dei microsismi generati da cicloni nel Mediterraneo. A questi corrisponde la prima parte della Tabella e della fig. 1 , fino ai periodi di 4 sec. In questo caso il rapporto $A_{\mathrm{r}} / A_{\mathrm{t}}$ è sensibilmente maggiore del valoro medio 2,1 sopra trovato. Questa maggiorazione $\dot{e}$, almeno in parte, facilmente spiegabile col fatto che Roma si trova proprio nel centro del Mediterraneo, quindi in genere sempre più vicina di Trieste al centro del ciclone, ed inoltre col fatto che i microsismi vi arrivano direttamente, senza dover superare discontinuità geologiche o tettoniche, o dover attraversare zone dalla tettonica molto movimentata, e quindi subirne cospicue dispersioni di energia.

Il massimo si ha intorno ai 3,5 sec di periodo: a questo valore sembrereblye corrispondere quindi una particolare risonanza locale, il

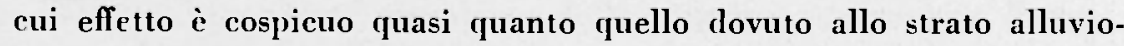
nale superficiale.

3. Casi anomali. - Vale la pena ora di menzionare i casi che possiamo chiamare anomali, in quanto si scostano dai valori normali 
sopraindicati. Per questi casi, i microsismi sono stati controllati sui sismogrammi originali sia di Trieste che di Roma, e la situazione meteorologica in corrispondenza è stata ricavata dalle carte sinottiche giornaliere del tempo publblicate dall'Lfficio Centrale Meteorologico Sivizero.

a) Casi di microsismi di lontana origine registrati „ Roma e non a Trieste.

Ne menzioniamo i principali, schematizzati e ordinati in ordine cronologico:

16 aprile 1948 - Leggero minimo a $\mathrm{S}$ costa Groenlandia, altro a N Norvegia; fronte freddo su Atlantico: Roma 4,0 sec, $1,0 \mu$.

17 aprile 1948 - Fronte treddo dalla Norvegia a $\mathbb{W}$ della Spagna (tutto su Atlantico); minimo leggero sul Golfo di Guascogna, con propaggini sul Mediterraneo occid.: Roma, $3.8 \mathrm{sec}, 2,2 \mu$.

18 aprile 1948 - Minimo fra Irlanda e Inghilterra; Roma $3,0 \mathrm{sec}, 1,6 \mu$.

19 aprile 1948 - Minimo a SW Golfo di Biscaglia: Roma 3,3 sec, $1,2 \mu$.

20 aprile 1948 - Minimo su mare del Nord, altro a W Golfo di Biscaglia: Roma $3,5 \mathrm{sec}, 1,0 \mu$.

24 aprile 1948 - Minimo su costa Groenlandia, fronti freddi su Atlantico e mare del Nord: Roma 3,6 sec, 1,8u.

26 aprile 1948 - Minimo a N Islanda: Roma $3,9 \mathrm{sec}, 1,4 \mu$.

I maggio 1948 - Minimo su Irlanda e mare adiacente, fronte freddo e occluso su Atlantico occid.: Roma 3,7 sec, $2,0 \mu$.

4 giugno 1948 - Minimo a S Islanda, altro minimo sul mare del Nord: Roma 4,0 sec, $3,2 \mu$.

9 giugno 1948 - Minimo su Atlantico: Roma 4,0 sec, $1,2 \mu$.

10 giugno 1948 - Immutato il minimo Atlantico: Roma 4,0 sec, $1,0 \mu$.

11 giugno 1948 - Permane il minimo del giorno 9.6: Roma 4,0 sec, 1,0 $\mu$. Tale continua a rimanere sempre nella stessa zona atlantica $(\mathrm{SW}$ Irlanda) fino al giorno 16 giugno, mantenendo quasi per tutio il periodo costante sia l'area depressionaria che il gradiente. A Trieste non e registrato, a Roma il periodo staziona sui 4,0 sec e l'ampiezza intorno a $1,0 \mu$.

Dal 17 giugno il ciclone si sposta leggermente verso $N$, ciò̀ a $N W$ dell'lrlanda. Trieste continua a non segnalare microsismi, mentre a Roma le loro ampiezze si riducono a circa $0,3 \mu$.

21 giugno 1948 - Ciclone a S Islanda: Roma, 4,0 sec, 2,2!.

7 luglio 1948 - Minimo su Scandinavia merid.; altro su Iberia e costa Atlantica: Roma 5,0 sec, $1,9 \mu$.

11 agosto 1948 - Minimo a S Islanda, fronte freddo su Atlantico: Roma $3,8 \mathrm{sec}$, $1,4 \mu$.

14 agosto 1948 - Minimo W Irlanda: Roma 4,0 sec, 3,1 $\mu$.

15 agosto 1948 - Minimo a N Irlanda: Roma 3,6 sec, $1,0 \mu$.

5 settemb. 1948 - Minimo a $\mathrm{SW}$ Irlanda: Roma $4,0 \mathrm{sec}, 1,6, \mathrm{l}$.

11 ottobre 1948 - Minimo a SW Islanda: Roma 5,0 sec, $0,9 \mu$.

12 ottobre 1948 - Minimo a W Islanda: Roma 6,0 sec, $0,8 \mu$. 
13 ottobre 1948 - Ciclone su Islanda: Roma 5,0 sec, 0,6

14 ottobre 1948 - Minimi su Jan Mayen e costa norvegese, quattro fronti freddi su Atlantico orient.: Roma 5,0 sec, $0,7 \mu$.

16 ottobre 1948 - Due minimi, uno a W Islanda, l'altro a W coste norvegesi : Roma 5,0 sec, $0,6 u$.

13 novemb. 1948 - Minimo a S Groenlandia, fronte occluso dal minimo verso S: Roma 5,0 sec, 0,511 .

15 novemb. 1948 - Minimo su Groenlandia, fronte occluso da esso fino W Spagna; segnalato a Roma alle $6^{\text {h }}$ con 3,5 sec, 0,4 ; alle $12^{\text {h }}$ con 3,5 sec, 1,5 i; Trieste: debolissimi solo alle $6^{\mathrm{t}}$.

18 dicemb. 1948 - Minimo a N Islanda. fronti freddi su tutto l'Atlantico orient.: Roma 5,0 sec, 1,0 !.

Come si vede, in questi casi il periodo dei microsismi a Roma è in prevalenza prossimo ai 3,5 sec sopra menzionati. Questa è un'altra prova di quanto abbiamo sopra detto $(\$ 2, b)$ sulla probabile risonanza del sottosuolo di Roma per onde sinusoidali con periodo di quest'ordine di grandezza. A Trieste in corrispondenza la "soglia microsismica " non viene varcata, cioè l'ampiezza dei microsismi rimane inferiore alla sensibilità strumentale, che è di 0,1 \%.

Interessante è ancora notare che in tutti i casi ora menzionati il periodo a Roma dovrebbe essere maggiore, di $6 \div 10 \mathrm{sec}(4)$. Ciò po. trebbe forse significare cbe neanche a Roma le onde di questi periodi riescono a diventare apprezzahili, ma che invece vengono straordinariamente esaltate solo quelle attorno ai 3,5 sec sopra menzionati.

b. Casi in cui il rapporto delle ampiezze fra microsismi di lontana origine registrati a Roma ed a Trieste è notevolmente maggiore della media normale.

Questi casi, che pure riportiamo qui schematizzati ed in ordine cronologico, diventano ora interpretabili alla luce di quanto esposto in $a$ ). Si osservi infatti che i periodi sono dell'ordine di $3 \div 5$ sec, cioè prossimi a quelli che producono risonanza a Roma: è da intendersi allora che, rispetto ai casi menzionati in $a$ ), l'intensità microsismica sia ora maggiore, tanto da produrre registrazioni apprezzabili anche a Trieste: a Roma poi, sempre per l'effetto della risonanza qui discussa, le ampiezze vengono notevolmente esaltate.

25 Jebbraio 1948 - Minimo su Groenlandia (quasi tutto su terra); altro minimo a W Spagna; fronte orcluso dalla Groenlandia all'Inghilterra: Roma 5,0 $\mathrm{sec}$, $5,7 \mu$; Trieste: 6,0 sec, $0,3 \mu$.

29 Jebbraio 1948 - Minimo S Groenlandia; fronte occluso dall'Inghilterra alla Norvegia: Roma 5,0 sec, $3,0 u$; Trieste 6,0 sec, $0,5 u$. 


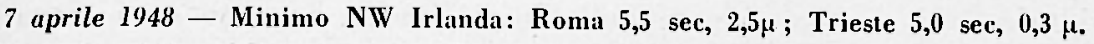
28 aprile 1948 - Minimo su Scandinavia, fronti freddi su mare del Nord e Atlantico: Roma 4,0 sec, $1,3 \mu$; Trieste $5,0 \mathrm{sec}, 0,2 \mathrm{u}$.

5 giugno 1948 - Minimo S Islanda: Romi 4,0 sec, 2,5 ; Trieste 4,0 sec, $0,4 \mu$.

o gıugno 1948 - Minimo fra Islanda e Irlanda: Roma 5,0 sec, 3,0 4 ; Trieste 5,0 sec, $0,4 \mu$.

7 giugno 1948 - Minimo N Inghilterra: Roma 4,0 sec, 1,3 1 ; Trieste 4,5 sec, $0,2 \mu$.

8 giugno 1948 - Tre deboli minimi su Atlantico occid., fronte freddo W Spagna: Roma $4,0 \mathrm{sec}, 1,3 \mu$; Trieste $4,0 \mathrm{sec}, 0,1 \mu$.

13 agosto 1948 - Minimo su mare del Nord: Roma 4,3 sec, 3,i $\mu$; Trieste 4,0 sec, $0,3 \mu$.

9 novembre 1948 - Minimo S Islanda, altro minimo su Atlantico W Irlanda: Roma 4,5 sec, 2,0 $\mu$; Trieste debolissimi.

11 novembre 1948 - Ciclone IV Irlanda, fronte freddo e occluso su Atlantico occid.: Roma 6,0 sec, $2,2 \mu$; Trieste 5,0 sec, $0,4 \mu$.

17 novembre 1948 - Minimo su Islanda: Roma 5,0 sec, $2,6 \mu$; Trieste 6,0 sec, $0,5 \mu$.

20 novembre 1948 - Minimo fra Islanda e Inghilterra: Roma 0,0 sec, $2,2 \mu$; Trieste 6,0 sec, $0,4 \mu$.

22 novembre 1948 - Ciclone su Atlantico (W Golfo di Biscaglia): Roma 5,5 sec, $1,4 \mu$; Trieste $5,5 \mathrm{sec}, 0,3 \mu$.

23 novembre 1948 - Ciclone S Groenlandia: Roma, 5,5 sec, 2,0 $\mu$; Trieste 5,5 sec, $0,4 \mu$.

27 novembre 1948 - Doppio ciclone fra Groenlandia e Islanda: Roma 5,0 sec, $1,3 \mu$; Trieste 5,0 sec, $0,1 \mu$.

28 novembre 1948 - Ciclone su Groenlandia, altro su Atlantico (W Golfo di Biscaglia): Roma 5,0 sec, $1,4 \mu$; Trieste 5,0 sec, $0,2 \mu$.

5 gennaio 1949 - Ciclone S Islanda: Roma 4,0 sec, 2,5 $\mu$; Trieste 5,0 sec, $0,3 \mu$.

6 gennaio 1949 - Il ciclone precedente si sposta a NE (tra Groenlandia e Norvegia); altro ciclone avanza nell'Atlantico a W Irlanda: i micros. sia a Roma che a Trieste sono in leggero aumento.

7 gennaio 1949 - Ciclone a NW Irlanda, fronte freddo su Atlantico: Roma 6,0 sec, $4,5 \mu$; Trieste $7,0 \mathrm{sec}, 0,8 \mu$.

16 gennaio 1949 - Ciclone $N$ Jan Mayen: Roma 4,0 sec, 1,5 $\mu$; Trieste quasi nulli.

13 gennaio 1949 - Minimo tra Groenlandia e Islanda: Roma 5,0 sec, 3,6 $\mu$; Trieste $6,0 \mathrm{sec}, 0,4 \mu$.

14 gennaio 1949 - Ciclone W Norvegia, fronti freddi su Islanda e mare del Nord: Roma 4,0 sec, 3,4 4 ; Trieste 5,5 sec, $0,4 \mu$.

2 febbraio 1949 - Minimo su Atlantico (W Spagna): Roma 4,5 sec, 2,3 $\mu$; Trieste 4,5 sec, 0,4 u.

3 febbraio 1949 - Minimo S Islanda, altro molto a W Irlanda; fronti freddi su Atlantico: Roma $4,0 \mathrm{sec}, 2,2 \mu$; Trieste 4,0 sec, $0,4 \mu$.

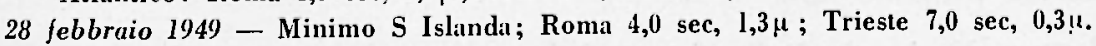

c) Casi in cui Trieste registra microsismi di lontana origine e Roma no. Per quanto si è visto sopra, i casi qui cercati sarebbero i piu 
interessanti, se sicuramente individuati. Invece ne sono stati trovati soltanto i due seguenti:

4 aprile 1948 - Due minimi: uno a NW Scozia, altro a $\mathrm{N}$ tra Islanda e Norvegia: Trieste $5,0 \mathrm{sec}, 0,4 \mu$; Roma $2,0 \div 3,0 \mathrm{sec}, 0,6 u$.

4 febbraio 1949 - Minimo su Atlantico (W Islanda): Trieste 7,5 sec, 1,0 i; Roma $3,5 \div 4,0$ sec, $2,0 \mu$.

Essi, come si vede, non sono prohatori nel senso cercato, in quanto a Roma i microsismi non mancano, ma hanno periodo molto minore; esso è prossimo al periodo di risonanza sopra individuato, e difatti le ampiezze sono a Roma maggiori.

4. Conclusioni. - Da quanto precede risulta chiaramente accertata anzitutto la notevole infuenza che sulle ampiezze e sui periodi dei microsismi registrati esercitano le condizioni geologiche locali, in particolare il sottosuolo superficiale in corrispondenza della Stazione. Per Roma, esso è tale da esaltare notevolmente i microsismi con periodo attorno ai 3,5 sec. Anzi, questo effetto di risonanza ̀̀ così cospicuo da alterare completamente la caratteristica principale dei microsismi ( ${ }^{1}$ ), di avere cioè periodi (e forma) caratteristici per ogni determinata zona di provenienza, e generalmente crescenti con la distanza.

La situazione di Roma risulta quindi ideale per lo studio dei microsismi, soprattutto di origine mediterranea, ed in particolare per studiare e seguire attraverso di essi la genesi, evoluzione e spostamenti dei centri di perturbazione atmosferica. Questo studio non si potrà però forse garantire pienamente con i dati di una sola Stazione, per gli effetti sopra menzionati della risonanza. Ma è da prevederne il massimo rendimento con l'impiego di una Stazione tripartita, specie se ogni vertice consisterà di una serie completa di tre sismografi, per eliminare l'effetto interferenziale $\left({ }^{5}\right)$ dovuto al fatto che generalmente si tratta di onde con periodi prossimi provenienti da diverse direzioni $\left(^{3}\right)$.

Facendo astrazione da questo effetto di risonanza dovuto al sottosuolo di Roma, i periodi dei microsismi dovuti a cicloni lontani sono in generale identici a Roma ed a Trieste: rientrando così nella norma prevista dalla teoria $\left({ }^{1}\right)$.

Istituto Nazionale di Geofisica - Osservatorio di Trieste - gennaio 1950. 


\section{RIASSUNTO}

I microsismi registrati in corrispondenza a Roma ed a Trieste vengono confrontati nei loro elementi caratteristici, periodo ed ampiezza. Risulta anzitutto che $i$ microsismi di lontana origine sono a Roma sempre più ampi, per un fattore di amplificazione medio dellordine di 2.1, in conseguenza dello strato alluvionale su cui poggia la Stazione. A questo si aggiunge un effetto di risonanza molto cospicuo per periodi attorno ai $3,5 \mathrm{sec}$. Infine per i microsismi di origine mediterranea si hanno a Roma le massime ampiezze. Ne consegue l'importanza della posizione di Roma per lo studio dei microsismi, nel quale però occorreranno particolari attenzioni per ridurre l'influsso dell'alterazione dovuta all'eccitabilità locale.

\section{BIBLIOGRAFIA}

(1) Morelli C.: Contributo allo studio dei microsismi. Annali di Geofisica, I, 4, 621-652, Roma 1948.

(-) D’Henry G.- Morelli C.: Sulle cause dei microsismi. Ibidem, II, 281-289, Koma 1949.

(3) D'Henry G.: Sulla natura fisica dei microsismi. Ibidem, III, 1, Roma 1950.

(4) Glorgi M.: Su alcuni aspetti caratteristici dei microsismi a Roma. Ibidem, II, 24-39, Roma 1949.

(5) DoN LeET L.: Discussion of tripartite microseismic measurements. Bull. Seismol. Soc. of America, 39, 4, 249.255, Oct. 1949. 